\title{
Transcranial direct current stimulation for the treatment of tinnitus: a review of clinical trials and mechanisms of action
}

Tifei Yuan ${ }^{1,2}$, Ali Yadollahpour ${ }^{3 *}$ D, Julio Salgado-Ramírez ${ }^{4}$, Daniel Robles-Camarillo ${ }^{5}$ and Rocío Ortega-Palacios ${ }^{4}$

\begin{abstract}
Background: Tinnitus is the perception of sound in the absence of any external acoustic stimulation. Transcranial direct current stimulation (tDCS) has shown promising though heterogeneous therapeutic outcomes for tinnitus. The present study aims to review the recent advances in applications of tDCS for tinnitus treatment. In addition, the clinical efficacy and main mechanisms of action of tDCS on suppressing tinnitus are discussed.

Methods: The study was performed in accordance with the PRISMA guidelines. The databases of the PubMed (1980-2018), Embase (1980-2018), PsycINFO (1850-2018), CINAHL, Web of Science, BIOSIS Previews (1990-2018), Cambridge Scientific Abstracts (1990-2018), and google scholar (1980-2018) using the set search terms. The date of the most recent search was 20 May, 2018. The randomized controlled trials that have assessed at least one therapeutic outcome measured before and after tDCS intervention were included in the final analysis.

Results: Different tDCS protocols were used for tinnitus ranging single to repeated sessions (up to 10) consisting of daily single session of 15 to 20-min and current intensities ranging 1-2 mA. Dorsolateral prefrontal cortex (DLPFC) and auditory cortex are the main targets of stimulation. Both single and repeated sessions showed moderate to significant treatment effects on tinnitus symptoms. In addition to improvements in tinnitus symptoms, the tDCS interventions particularly bifrontal DLPFC showed beneficial outcomes on depression and anxiety comorbid with tinnitus. Heterogeneities in the type of tinnitus, tDCS devices, protocols, and site of stimulation made the systematic reviews of the literature difficult. However, the current evidence shows that tDCS can be developed as an adjunct or complementary treatment for intractable tinnitus. TDCS may be a safe and cost-effective treatment for tinnitus in the short-term application.
\end{abstract}

Conclusions: The current literature shows moderate to significant therapeutic efficacy of tDCS on tinnitus symptoms. Further randomized placebo-controlled double-blind trials with large sample sizes are needed to reach a definitive conclusion on the efficacy of tDCS for tinnitus. Future studies should further focus on developing efficient disease- and patient-specific protocols.

Keywords: Transcranial direct current stimulation, Tinnitus, Treatment, Clinical efficacy

\footnotetext{
*Correspondence: Yadollahpour.a@gmail.com

${ }^{3}$ Department of Medical Physics, School of Medicine, Ahvaz Jundishapur

University of Medical Sciences, Golestan Blvd, Ahvaz 61357-33118, Iran

Full list of author information is available at the end of the article
}

C The Author(s) 2018. This article is distributed under the terms of the Creative Commons Attribution 4.0 International License (http://creativecommons.org/licenses/by/4.0/), which permits unrestricted use, distribution, and reproduction in any medium, provided you give appropriate credit to the original author(s) and the source, provide a link to the Creative Commons license, and indicate if changes were made. The Creative Commons Public Domain Dedication waiver (http://creativecommons.org/ publicdomain/zero/1.0/) applies to the data made available in this article, unless otherwise stated. 


\section{Background}

Tinnitus is the perception of sound, in the ear or in the head, in the absence of any external acoustic stimulation which affects $10-15 \%$ of the adult population worldwide $[1,2]$. The main risk factors of tinnitus include hearing loss, trauma to the auditory periphery such as a lesion to auditory nerve, abnormal plastic changes in auditory network, ototoxic medications, head injury, and depression [3]. Hearing loss is not necessarily a precondition of tinnitus; however, some studies have shown that different forms of hearing loss may have correlation with tinnitus $[2,4]$. This disorder is usually accompanied by different mild to severe comorbidities such as depression, anxiety, and sleep disturbances that make it a debilitating condition $[1,2,5]$.

Neuroimaging, neuroelectrophysiologic, and neuroanatomic studies have shown that maladaptive plastic changes in different auditory and non-auditory cerebral regions and abnormal neural activities of specific cortical regions might be the main etiology of tinnitus [6-10]. Tinnitus perception is an integrated output of a large and complicated brain network comprising of different subnetworks with overlapping functions [2, 11]. In this impaired network, each subnetwork represents a clinical aspect of tinnitus such as distress, loudness, and laterality [11-13]. Neurobiological and neuroimaging findings have shown that abnormal activities of the non-auditory regions associated with cognitive and attentional functions as well as limbic processes probably contribute to the unpleasant and distressing aspects of tinnitus [11, 14]. Structural and functional abnormalities in dorsolateral prefrontal cortex (DLPFC) [10, 15-17] and auditory cortex (AC) $[2,3,18,19]$ are associated with tinnitus. The DLPFC is a multifunction region that plays important roles in auditory processing and perception, auditory attention, top-down modulation of auditory processing, and modulating the input to primary AC [20-22]. Moreover, DLPFC is involved in regulating different cognitive functions. Therefore, in development of any treatment modality for tinnitus, this disorder should be considered as a complex and heterogamous condition involving a large network consisting of multiple overlapping brain networks. Considering the engagements of $\mathrm{AC}$ and DLPFC in the tinnitus perception, these regions may be good targets for any therapeutic intervention for tinnitus.

Several pharmacologic agents have been developed for tinnitus treatment; however, a large portion of the patients are resistant to the treatment [1]. In addition, most of the pharmacologic drugs are associated with different side effects that adversely influence the individual's daily and quality of life. So far, no definitive treatment has been proposed for tinnitus and several common causes of tinnitus remain elusive. In this regard, studies are ongoing to develop new efficient therapeutic modalities for tinnitus in two avenues including pharmacologic agents and non-pharmacologic modalities.

During the recent decades, several non-pharmacological modalities such as cognitive behavioral therapies [23], noise-masking modality [24], and neurofeedback [25] have been proposed for treatment of tinnitus; however, they have limited treatment efficacy and each of them have their own drawbacks.

Applications of brain stimulation and modulation techniques have been dramatically developed during the recent decade for treatment and management of neuropsychiatric disorders [26-31]. These modalities including repetitive transcranial magnetic stimulation (rTMS), deep brain stimulation, and electrical stimulation have shown promising outcomes in the disorders in which the abnormal neural activities and impaired neural interfaces are the main characteristics [26-30]. This significant contribution of neural stimulation and modulation modalities is mostly because of mutual interactions between the endogenous and exogenous electrical and magnetic fields. The therapeutic values of electric and magnetic fields have been reported in different disorders that support the above claim [32-35].

RTMS has shown therapeutic effects in tinnitus through eliminating the tinnitus symptoms and also improving the cognitive impairments comorbid with tinnitus [36-38]. However, this technique is relatively expensive and associated with side effects with lower mobility.

Transcranial direct current stimulation (tDCS) is a form of neuromodulation in which a low intensity direct current passes the brain tissues through a pair of electrodes placed on the scalp. The tDCS is a noninvasive, safe, cost-effective, and user friendly modality which has shown promising outcomes in treatment of different neuropsychiatric disorders as well as in improving cognitive functions in healthy individuals [39-43].

Tinnitus is associated by abnormal neural activities in different brain regions and also maladaptive neuroplasticity of specific regions. Therefore, tDCS applied over specific brain regions with appropriate anodal/cathodal placement has been expected to have beneficial effects for this disorder. Anodal and cathodal tDCS respectively increases (depolarizes) and reduces (hyperpolarizes) the cortical excitability of the exposed regions [44].

Several preclinical and clinical studies have been conducted on tinnitus and the initial findings were promising though controversial [45-48]. Studies are ongoing to reach a definitive answer on the clinical efficacy of tDCS in tinnitus.

Studies are ongoing to develop effective clinical protocols and to understand the mechanisms of action. 
The present study aims to review the recent advances in applications of $\mathrm{tDCS}$ for tinnitus treatment. In addition, the clinical efficacy and the main mechanisms of action of the technique are discussed.

\section{Methods}

The databases of the PubMed (1980-2018), Embase (1980-2018), PsycINFO (1850 -2018), CINAHL, Web of Science, BIOSIS Previews (1990-2018), Cambridge Scientific Abstracts (1990-2018), and google scholar (1980-2018) using the set search terms. The study procedures were performed according to the guidelines of the PRISMA. The search terms were "transcranial direct current stimulation" OR "tDCS” AND "tinnitus" AND "treatment". The date of the most recent search was 20 May 2018. Bibliographies of the retrieved records and review articles were manually reviewed to identify the records that may have been missed in the initial search. The titles, abstracts, and keywords of all retrieved records were reviewed and the eligible records were entered in the final review based on the inclusion and exclusion criteria. Only published, peer-reviewed studies on human subjects available in English were considered for this review. The studies that investigated the treatment of different types of tinnitus with different tDCS devices and in different protocols against sham condition were included in the review. Studies of randomized controlled trials were included.

The studies should have assessed at least one therapeutic outcome measured before and after an intervention. The studies that assessed only cognitive measures, studies on animal and healthy subjects were also excluded. Clinical trials without a randomized controlled design, conference abstracts, narrative reviews, and editorials were excluded from the review.

\section{Results}

A total of 85 studies were identified at the screening step. In the identification phase, total of 33 records were excluded from the further assessment and 53 records were entered into the screening phase in which 8 conference abstracts, 1 book, 2 case reports and 4 editorials were excluded from the review. In the eligibility stage, 31 records remained in the study and 3 records from the additional records were added into the study where total of 34 studies were included for detailed review. Due to the heterogeneities in the patients and the tDCS devices and protocols as well as the target sides, the authors decided to comprehensively review the studies. The review focuses on the advances in applications of tDCS for treatment of tinnitus and the important factors in the resulting outcomes. In addition, the mechanisms of actions of the tDCS in tinnitus treatment are discussed (Fig. 1).

\section{Discussion}

Tinnitus is a heterogeneous disorder in which several regions are involved in the tinnitus-related anomalies ranging primary and secondary auditory systems as well as non-auditory brain areas.

The general hypothesis in application of tDCS for treatment of tinnitus like other neuropsychiatric disorders is that anodal tDCS increases the neural excitability, whereas cathodal tDCS decreases it. As a result: anodal tDCS with excitatory effect can be applied on the regions with hypo-activity associated with an impairment to reach beneficial outcome. Similarly, cathodal tDCS that induces inhibitory effect can be applied over the regions with disease specific hyper-activities to reach beneficial effects.

The main approach in choosing the target site and electrode placement in tDCS applications in different neuropsychiatric disorders is modulating the impaired region(s) of the brain to alter the activities or functions of the region(s) towards normal conditions. In this regard, for tinnitus, the main objective is modulating either the tinnitus percept or its affective aspects like distress through disrupting the underlying pathological neural activities. The general hypothesis is that anodal tDCS increases the neural excitability, whereas cathodal tDCS decreases it [44, 49-51]. As a result: anodal tDCS with excitatory effect can be applied on the regions with hypoactivity associated with an impairment to reach beneficial outcome [52]. Similarly, cathodal tDCS that induces inhibitory effect can be applied over the regions with disease specific hyper-activities to reach beneficial effects [52].

Considering the tinnitus features and the associations between tinnitus and the structural and functional abnormalities in DLPFC [10, 15-17] and AC [2, 3, 18, $19]$, these two sites were the main targets in the previous tDCS studies in tinnitus. Initial tDCS studies have targeted the $\mathrm{AC}$ for tinnitus treatment and the findings were promising though controversial [53-55]. Moreover, modulating DLPFC activity using tDCS has been shown to enhance different cognitive functions in healthy individual and to improve different neuropsychiatric disorders, including tinnitus [17, 39, 40, 56-58].

The two main sites targeted in the previous studies for treatment of tinnitus were DLPFC $[13,59,60]$ and AC $[45,54,61]$. Fregni et al. conducted the first study investigating tDCS in tinnitus in which they compared the effects of cathodal tDCS, anodal tDCS, and $10-\mathrm{Hz}$ rTMS against sham stimulation over two sites of mesial parietal cortex and left temporoparietal area (LTA) 


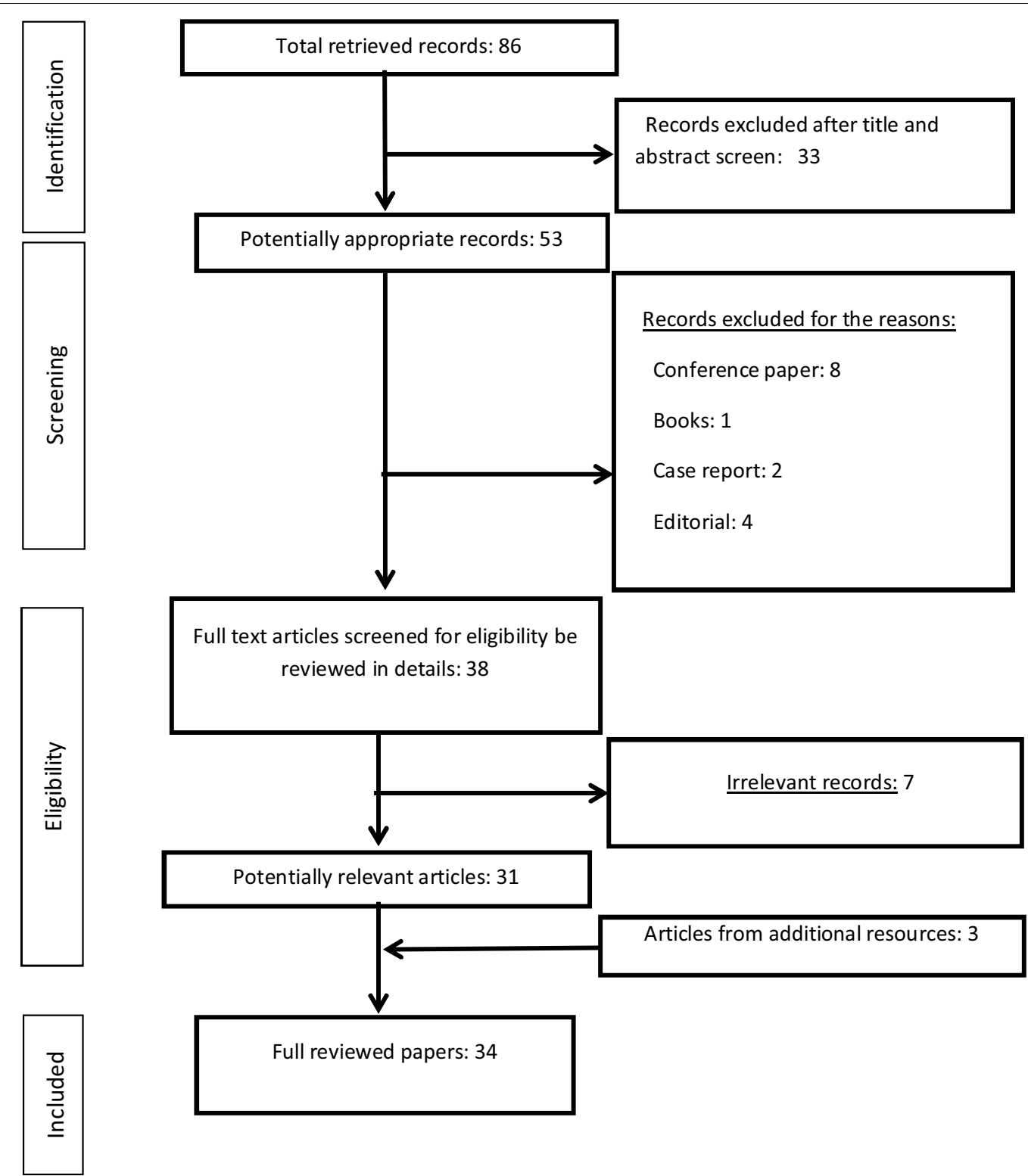

Fig. 1 The PRISMA flow chart of the study

on tinnitus symptoms [45]. They reported that $10 \mathrm{~Hz}$ rTMS and anodal tDCS of LTA significantly reduced the tinnitus symptoms; however, the effect was transient and short lasting. After this study, several clinical trials have been conducted to evaluate and develop the therapeutic efficacy of tDCS for treatment of tinnitus. Garin et al. investigated the outcomes of tDCS applied over LTA and reported significant improvements in tinnitus symptoms and interestingly they reported the beneficial effects lasted for several days in some patients [62]. Both Fregni et al. and Garin et al. reported that cathodal tDCS over the LTA with the anode on the contralateral supraorbital area did not improve tinnitus symptoms.

Following these initial studies, other researchers have investigated the tDCS in single and repeated sessions over LTA and AC and reported controversial findings $[54,63,64]$. It seems that cathodal tDCS in single session is not effective on tinnitus treatment since cathodal tDCS is not strong enough to disturb or modulate the ongoing tinnitus-related abnormal cortical activities [65]. In this regard, repeated sessions of cathodal tDCS may have therapeutic effects on tinnitus based on the theoretical and experimental results. Therefore, repeated sessions 
of tDCS, longer period of each session and higher intensities have been designed to investigate the effects of cathodal tDCS in tinnitus [54, 60, 66, 67].

Following the initial studies focusing on temporoparietal area (TA) and $\mathrm{AC}$, several studies have targeted prefrontal cortex (PFC), particularly DLPFC for tinnitus treatment. In these studies, the main target site was DLPFC and the most frequent electrode montage was bifrontal $[13,46,59,68]$. Vanneste et al. were the first group reported the effects of bifrontal tDCS on tinnitus symptoms [13]. They investigated the effects of bifrontal tDCS over DLPFC $(n=478)$ in an open label study. They applied bifrontal tDCS ( $2 \mathrm{~mA}$, each session $20 \mathrm{~min})$ in two montages (anode right/cathode left $(\mathrm{n}=448)$ and anode left/cathode right $(\mathrm{n}=30)$ DLPFC) for $20 \mathrm{~min}$. They reported no tinnitus-suppressing effect in the anode left/cathode right DLPFC tDCS. However, anode right/cathode left tDCS significantly reduced the tinnitus intensity or distress in $29.9 \%$ of the patients. In addition, they observed an interaction between the amount of distress reduction and the tinnitus laterality. However, they did not observe such interaction for the tinnitus intensity [13]. Vanneste et al. concluded that bifrontal tDCS could modulate the emotional aspects of tinnitus experienced by the patients [13].

The next studies conducted on bifrontal tDCS have reported that this montage both in anode left/cathode right or vice versa could improve the tinnitus-related depression and anxiety, respectively [47]. These effects could be attributed the roles of PFC and particularly DLPFC in modulating different non-auditory structures and networks involved in perception of the auditory and distress aspects of tinnitus as well as emotional functions.

To improve the tDCS protocol in targeting the optimal stimulation site for tinnitus treatment, De Ridder and Vanneste $(n=675)$ compared the efficacy of EEGdriven tDCS versus standard bifrontal tDCS [61]. They used source localized resting-state electrical activity to determine gamma-band functional connectivity as an index of the tinnitus network. On the one hand, the authors reported that standard bifrontal tDCS, with the anode right/cathode left DLPFC, significantly reduced tinnitus symptoms in $30 \%$ of the patients [61]. Moreover, the EEG-driven tDCS approach did not significantly improve the symptoms. The authors also tried to identify the mechanism of action of tDCS in suppressing the tinnitus symptoms through comparing the pre- and post-intervention of the source-localized resting-state electrical activity of the patients. They concluded that the tDCS induced changes are probably occurred through modulations of a large network consisting of pregenual anterior cingulate cortex, parahippocampal area, and right primary $\mathrm{AC}$ in resting-state spontaneous brain activity. This study demonstrated that tDCS impacts both the direct target under the electrodes (DLPFC) and distant regions with functional connections with the exposed target [61]. This finding along with neuroimaging studies encourage further studies on the therapeutic outcomes of tDCS applied over non-auditory regions in tinnitus.

In line with the optimization of $\mathrm{tDCS}$ protocol for tinnitus, Shekhawat and Vanneste designed a trial to optimize the parameters of bifrontal tDCS over DLPFC for tinnitus suppression with the primary outcome of tinnitus loudness [68]. They designed a dose-response trial $(n=111)$ to optimize the current intensity (1.5 and $2 \mathrm{~mA}$ ), stimulation duration ( 20 and $30 \mathrm{~min}$ ), and number of tDCS sessions $(2,4,6,8$, and 10 with 3-4day washout period between each session). The patients received a minimum of 2 sessions during 1 week or maximum of 10 sessions during 5 weeks. Their findings showed a significant reduction in tinnitus loudness after DLPFC tDCS. The intensity and duration of each session did not show significant interaction with the outcome. In addition, they reported that increasing the number of sessions increases the amount of outcome, but after 6 sessions no further increase was observed and the amount of outcome reached a plateau trend [68].

Few studies have used different electrode montages than the previous studies triggering LTA or AC and DLPFC. For instance Pal et al. in a randomized, parallel, double-blind, sham-controlled study investigated the treatment efficacy and safety of cathodal tDCS to the AC with anode over the PFC [69]. They applied a 5-session tDCS over five consecutive days and assessed the tinnitus handicap inventory (THI) score as the primary outcome of tinnitus after the last session on day 5 , and at 1 and 3 months post stimulation. They reported no beneficial effects of tDCS on the neither primary nor secondary outcome measures. Their findings showed that tDCS of the auditory and prefrontal cortices does not improve tinnitus but it is relatively safe protocol [69].

A line of studies have focused on combinations of tDCS with other treatment modalities including pharmacological and non-pharmacological modalities [63, 70]. Shekhawat et al. in a 7-month long double-blind randomized clinical trial investigated the effects of multisession anodal tDCS over LTA combined with the hearing aid sound therapy in patients with chronic tinnitus $(\mathrm{n}=40)$. They applied anodal tDCS $(2 \mathrm{~mA}$ intensity; 20-min duration) for 5 consecutive sessions with 24-h gap over the LTA, and then applied a hearing aid treatment for 6 months. Their findings showed a significant improvement in the overall Tinnitus Functional Index score as well as the tinnitus loudness and distress scores. They reported that after 3 months of hearing aid use, 
significant improvements were observed in tinnitus that were sustained at 6 months of use [63].

Teismann et al. investigated the effects of combined tailor-made notched music training (TMNMT) with tDCS on tinnitus symptoms. They applied TMNMT for 10 subsequent days (daily single session of $2.5 \mathrm{~h}$ ). During the initial 30-min of the first 5 days of the TMNMT sessions, they concurrently applied tDCS (intensity: $2 \mathrm{~mA}$ ) in anodal, cathodal, and sham groups. The active electrode was over the head surface over left AC; the reference electrode was put over right supraorbital cortex [70]. They observed a significant reduction in tinnitus handicap inventory (THI) score that reached its maximum value after the 5 days of treatment. The treatment effect remained significant for 31 days following the termination of the treatment. They also reported no significant difference between the anodal, cathodal, or sham tDCS groups.

It seems that tDCS over TA or AC may have greater therapeutic effects when combined with other non-medication modalities.

So far, most of the tDCS trials for tinnitus treatment have investigated the effects of single session tDCS on tinnitus symptoms. However, few studies have assessed the treatment effects of repeated sessions of tDCS on tinnitus symptoms. In these studies the number of total sessions ranged three to ten sessions consisting daily one session and current intensity ranging $1-2 \mathrm{~mA}$ and each session lasting 15 to $30 \mathrm{~min}$. The main target sites in the tDCS applications in tinnitus treatment were temporal or temporoparietal (auditory) cortex [48, 54, 71, 72] and DLPFC [47, 55, 73]. The findings of these studies are promising though heterogeneous which encourage conducting further placebo-controlled randomized trials to shed more light on the clinical efficacy of the technique and mechanism of action involved in the effects. One important factor that should be further assessed in future studies is assessing the treatment outcomes for longer follow up periods since most of the previous studies have investigated the transient effects of $\mathrm{tDCS}$ and in few cases the after effect assessments were not beyond some hours.

Neuroimaging and neurobiological studies have demonstrated that the main features of tinnitus are hyperactivity and maladaptive plasticity in $\mathrm{AC}[2,6,9]$. In tinnitus there are specific neural changes that start at the cochlear nucleus and project to the AC and non-auditory brain regions. The main cause of these neural anomalies is maladaptive neural plasticity. This maladaptive plasticity increases spontaneous firing rates of and synchrony among neurons in primary and secondary auditory systems that may generate the phantom percept. In addition to the abnormal neural activities and maladaptive plasticity present in the primary and secondary
ACs, disturbances in non-auditory brain structures and networks such as the insula, anterior cingulate cortex, and the DLPFC have been proposed as other possible pathologies of tinnitus [6, 8-10, 13, 18, 74, 75]. The perception of tinnitus has been reportedly as an integrated output of a complex tinnitus network consisting of different regions and subnetworks. It is assumed that each subnetwork of this network represents a clinical aspect of tinnitus such as distress, loudness, laterality, etc.

There are different hypotheses proposed to explain the therapeutic outcomes of tDCS in tinnitus symptoms. The first hypothesis is based on the disturbing theory of an ongoing neural activity associated with tinnitus. It is hypothesized that tDCS disturbs the abnormal ongoing neural activity induced by tinnitus. The second hypothesis is changing the maladaptive plasticity of tinnitus through repeated sessions of tDCS. Previous studies have shown that repeated sessions of tDCS depending on the polarity of the electrode could reduce or increase the neural excitability of the exposed regions and the resulting changes persist beyond the tDCS intervention $[44,76]$. This altered excitability can lead to neuroplasticity with therapeutic effects for tinnitus [52]. Therefore, in the treatment of tinnitus with tDCS, the main idea is modulating the abnormal excitability in the auditory pathways and maladaptive plasticity in auditory and limbic cortexes through applying single or repeated sessions of tDCS. The clinical trials conducted so far have shown that single and repeated sessions of tDCS applied over DLPFC or AC may induce transient and long lasting therapeutic effects in tinnitus patients. The early studies have investigated the effects of single session tDCS and reported transient beneficial effect, but the effects did not last more than some hours.

Some evidence showed that the tDCS effects on tinnitus symptoms are probably induced through modulations of a large neural network comprising of pregenual anterior cingulate cortex, parahippocampal area, and right primary $\mathrm{AC}$ in resting-state spontaneous brain activity [61]. According to this hypothesis, the tDCS influences both the direct target under the electrodes and distant regions with functional connections with the direct target [61].

Most of the previous studies have investigated the physical parameters of tDCS to develop effective treatment protocols for tinnitus and also in other neuropsychiatric disorders including the electrode size, polarity, electrode placement and configuration, current amplitude and density, treatment duration, number of sessions and total dose. Findings of the recent studies as well as neuroimaging and neuroelectrophysiologic studies showed that tinnitus is a heterogeneous disease with different diseasespecific features. It seems that in addition to the physical 
parameters of $\mathrm{tDCS}$, the patient- and disease-specific factors including gender, audiometric variables, severity of tinnitus, tinnitus laterality and type, illness duration, and audiometric features of the patients might be important in exerting and/or the amount of therapeutic effects [47, $59,60]$. Therefore, at least one line of the future tDCS studies for treatment of tinnitus should focus on developing disease specific of $\mathrm{tDCS}$ protocols.

\section{Conclusions}

This study reviewed the advances in using tDCS for treatment of tinnitus and discussed the therapeutic efficacy of the technique and the main mechanisms of action in treatment of tinnitus symptoms and therapeutic effects. Reviewing the current clinical trials showed that tDCS has moderate and promising treatment outcomes in the treatment of tinnitus. In addition, tDCS has shown beneficial effects on different cognitive impairments comorbid with tinnitus including anxiety and depression. However, so far there is no standard tDCS protocol for tinnitus treatment for clinical applications.

The main limitations of the conducted trials are small sample size, heterogeneities in patients and treatment protocols, poor methodology design, as well as the heterogeneous nature of tinnitus.

To develop efficient tDCS protocols for tinnitus, the roles of specific features of patient and tinnitus such as audiometric features of the patients, tinnitus laterality, tinnitus type, and tinnitus duration should be evaluated as well as the effects of the stimulation parameters. Further prospective, randomized, placebo-controlled, double-blind studies with large sample sizes are needed to reach a definitive conclusion on the efficacy of tDCS for tinnitus patients. Future studies should focus on developing efficient disease- and patient-specific protocols.

\footnotetext{
Abbreviations

AC: auditory cortex; LTA: left temporoparietal area; PFC: prefrontal cortex; PRISMA: preferred reporting items for systematic reviews and meta-analyses; rTMS: repetitive transcranial magnetic stimulation; TA: temporoparietal area; tDCS: transcranial direct current stimulation; $\mathrm{THI}$ : tinnitus handicap inventory; TMNMT: tailor-made notched music training.
}

\section{Authors' contributions}

Conceptualization and design, TY and AY JS-R and DR-C and RO-P; Data acquisition, TY and AY and JS-R and DR-C and RO-P; Analysis and interpretation, TY and $A Y$ and JS-R and DR-C and RO-P; Writing-Original draft preparation, TY and DR-C and RO-P; Writing-Review \& editing, AY and JS-R; Approval of manuscript, TY and AY and JS-R and DR-C and RO-P. All authors read and approved the final manuscript.

\footnotetext{
Author details

${ }^{1}$ Shanghai Key Laboratory of Psychotic Disorders, Shanghai Mental Health Center, Shanghai Jiao Tong University School of Medicine, Shanghai, China. ${ }^{2}$ Co-innovation Center of Neuroregeneration, Nantong University, Nantong, Jiangsu, China. ${ }^{3}$ Department of Medical Physics, School of Medicine, Ahvaz Jundishapur University of Medical Sciences, Golestan Blvd, Ahvaz 61357-33118, Iran. ${ }^{4}$ Biomedical Engineering Department, Polytechnic
}

University of Pachuca, Zempoala, Mexico. ${ }^{5}$ Graduate and Research Department, Polytechnic University of Pachuca, Zempoala, Mexico.

\section{Acknowledgements}

Not applicable.

\section{Competing interests}

Ali Yadollahpour and Tifei Yuan are members of the editorial board for BMC Neuroscience. There is no other competing interest declared by authors.

\section{Availability of data and materials}

All data generated or analyzed during this study are included in this published article.

\section{Consent to publish}

Not applicable.

\section{Ethics approval and consent to participate}

Not applicable.

\section{Funding}

Authors received no fund for this study.

\section{Publisher's Note}

Springer Nature remains neutral with regard to jurisdictional claims in published maps and institutional affiliations.

Received: 27 June 2018 Accepted: 23 October 2018

Published online: 25 October 2018

\section{References}

1. Langguth B, Kreuzer PM, Kleinjung T, De Ridder D. Tinnitus: causes and clinical management. Lancet Neurol. 2013;12:920-30.

2. Baguley D, McFerran D, Hall D. Tinnitus. Lancet Lond Engl. 2013;382:16007. https://doi.org/10.1016/S0140-6736(13)60142-7.

3. Muhlnickel W, Elbert T, Taub E, Flor H. Reorganization of auditory cortex in tinnitus. Proc Natl Acad Sci USA. 1998;95:10340-3.

4. Husain FT, Schmidt SA. Using resting state functional connectivity to unravel networks of tinnitus. Hear Res. 2014;307:153-62.

5. Sindhusake D, Mitchell P, Newall P, Golding M, Rochtchina E, Rubin G. Prevalence and characteristics of tinnitus in older adults: the blue mountains hearing study: prevalencia y características del acúfeno en adultos mayores: el Estudio de audición blue mountains. Int J Audiol. 2003;42:289-94.

6. Schlee W, Hartmann T, Langguth B, Weisz N. Abnormal resting-state cortical coupling in chronic tinnitus. BMC Neurosci. 2009;10:11.

7. De Ridder D, Elgoyhen AB, Romo R, Langguth B. Phantom percepts: tinnitus and pain as persisting aversive memory networks. Proc Natl Acad Sci. 2011;108:8075-80.

8. Van Der Loo E, Congedo M, Vanneste S, Van De Heyning P, De Ridder D. Insular lateralization in tinnitus distress. Auton Neurosci. 2011;165:191-4.

9. Vanneste S, Van de Heyning P, De Ridder D. The neural network of phantom sound changes over time: a comparison between recent-onset and chronic tinnitus patients. Eur J Neurosci. 2011;34:718-31.

10. De Ridder D, Fransen H, Francois O, Sunaert S, Kovacs S, Van De Heyning P. Amygdalohippocampal involvement in tinnitus and auditory memory. Acta Otolaryngol. 2006;126:50-3.

11. Vanneste S, Plazier M, Van Der Loo E, Van de Heyning P, Congedo M, De Ridder D. The neural correlates of tinnitus-related distress. Neuroimage. 2010;52:470-80.

12. De Ridder D, Vanneste $S$, Congedo M. The distressed brain: a group blind source separation analysis on tinnitus. PLoS One. 2011;6:e24273.

13. Vanneste S, Plazier M, Ost J, van der Loo E, Van de Heyning P, De Ridder D. Bilateral dorsolateral prefrontal cortex modulation for tinnitus by transcranial direct current stimulation: a preliminary clinical study. Exp Brain Res. 2010;202:779-85.

14. Eggermont JJ. The neuroscience of tinnitus. Oxford: Oxford University Press; 2012 
15. Fitzgerald PB, Oxley TJ, Laird AR, Kulkarni J, Egan GF, Daskalakis ZJ. An analysis of functional neuroimaging studies of dorsolateral prefrontal cortical activity in depression. Psychiatry Res Neuroimaging. 2006;148:33-45.

16. Bodner M, Kroger J, Fuster JM. Auditory memory cells in dorsolateral prefrontal cortex. Neuroreport. 1996;7:1905-8.

17. Shekhawat GS, Stinear CM, Searchfield GD. Modulation of perception or emotion? a scoping review of tinnitus neuromodulation using transcranial direct current stimulation. Neurorehabil Neural Repair. 2015;29:83746. https://doi.org/10.1177/1545968314567152.

18. Rauschecker JP, Leaver AM, Mühlau M. Tuning out the noise: limbicauditory interactions in tinnitus. Neuron. 2010;66:819-26.

19. Schlee W, Schecklmann M, Lehner A, Kreuzer PM, Vielsmeier V, Poeppl $\mathrm{TB}$, et al. Reduced variability of auditory alpha activity in chronic tinnitus. Neural Plast. 2014. https://doi.org/10.1155/2014/436146.

20. Lewis JW, Beauchamp MS, DeYoe EA. A comparison of visual and auditory motion processing in human cerebral cortex. Cereb Cortex. 2000;10:873-88.

21. Mitchell TV, Morey RA, Inan S, Belger A. Functional magnetic resonance imaging measure of automatic and controlled auditory processing. Neuroreport. 2005;16:457.

22. Voisin J, Bidet-Caulet A, Bertrand O, Fonlupt P. Listening in silence activates auditory areas: a functional magnetic resonance imaging study. J Neurosci. 2006;26:273-8.

23. Bruder GE, Stewart JW, Mercier MA, Agosti V, Leite P, Donovan S, et al. Outcome of cognitive-behavioral therapy for depression: relation to hemispheric dominance for verbal processing. J Abnorm Psychol. 1997;106:138.

24. Shekhawat GS, Kobayashi K, Searchfield GD. Methodology for studying the transient effects of transcranial direct current stimulation combined with auditory residual inhibition on tinnitus. J Neurosci Methods. 2015;239:28-33.

25. Khoramzadeh S, Saki N, Davoodi I, Nosratabadi M, Yadollahpour A. Investigating the therapeutic efficacy of neurofeedback treatment on the severity of symptoms and quality of life in patients with tinnitus. Int J Ment Health Addict. 2016;14:982-92. https://doi.org/10.1007/s1146 9-016-9670-6.

26. Paulus W. Transcranial electrical stimulation (tES-tDCS; tRNS, tACS) methods. Neuropsychol Rehabil. 2011;21:602-17.

27. George MS, Wassermann EM, Williams WA, Callahan A, Ketter TA, Basser P, Hallett M, Post RM. Daily repetitive transcranial magnetic stimulation (rTMS) improves mood in depression. Neuroreport. 1995;6:1853-6.

28. Yadollahpour A, Rashidi S, Kunwar PS. Repetitive transcranial magnetic stimulation in psychiatric disorders: a review of clinical advances. Asian J Pharm. 2017;11:S242-50. https://www.scopus.com/inward/recor d.uri?eid $=2-s 2.0-85026263088 \&$ partnerlD $=40 \& \mathrm{md} 5=\mathrm{d} 1 \mathrm{da} 8 \mathrm{a} 4 \mathrm{~d} 7 \mathrm{df} 6 \mathrm{be} 4$ b49b98f6620ead12c. Accessed 11 June 2018.

29. Yadollahpour A, Jalilifar M, Rashidi S. Transcranial direct current stimulation for the treatment of depression: a comprehensive review of the recent advances. Int J Ment Health Addict. 2017;15:434-43. https://doi. org/10.1007/s11469-017-9741-3.

30. Lisanby $\mathrm{SH}$. Electroconvulsive therapy for depression. N Engl J Med. 2007;357:1939-45.

31. Lisanby S. Noninvasive brain stimulation for depression - the devil is in the dosing. N Engl J Med. 2017;376:2593-4.

32. Yadollahpour A, Jalilifar M, Rashidi S. Antimicrobial effects of electromagnetic fields: a review of current techniques and mechanisms of action. J Pure Appl Microbiol. 2014;8:4031-43. https://www.scopus.com/inward/ record.uri?eid=2-s2.0-84923860983\&partnerlD=40\&md5=4c6811 a94a 88290721c46a3a7d6c0c46. Accessed 11 June 2018.

33. Athanasiou A, Karkambounas S, Batistatou A, Lykoudis E, Katsaraki A, Kartsiouni T, et al. The effect of pulsed electromagnetic fields on secondary skin wound healing: an experimental study. Bioelectromagnetics. 2007;28:362-8.

34. McLeod KJ, Rubin CT, Donahue HJ. Electromagnetic fields in bone repair and adaptation. Radio Sci. 1995;30:233-44.

35. Yadollahpour A, Rashidi S. A review of electromagnetic field based treatments for different bone fractures. Biosci Biotechnol Res Asia. 2014;11:611-20. https://doi.org/10.13005/bbra/1313.

36. Kreuzer PM, Landgrebe M, ScheckImann M, Poeppl TB, Vielsmeier V, Hajak $\mathrm{G}$, et al. Can temporal repetitive transcranial magnetic stimulation be enhanced by targeting affective components of tinnitus with frontal rTMS? A randomized controlled pilot trial. Front Syst Neurosci. 2011;5:88.

37. Lefaucheur JP, Brugières P, Guimont F, Iglesias S, Franco-Rodrigues A, Liégeois-Chauvel C, et al. Navigated rTMS for the treatment of tinnitus: a pilot study with assessment by fMRI and AEPs. Neurophysiol Clin. 2012:42:95-109.

38. Langguth B, Eichhammer $\mathrm{P}$, Zowe $M$, Marienhagen J, Kleinjung T, Jacob $P$, et al. Low frequency repetitive transcranial magnetic stimulation (rTMS) for the treatment of chronic tinnitus-are there long-term effects? Psychiatr Prax. 2004;31:S52-4.

39. Yadollahpour A, Asl HM, Rashidi S. Transcranial direct current stimulation as a non-medication modality for attention enhancement: a review of the literature. Res J Pharm Technol. 2017;10:311-6. https://doi. org/10.5958/0974-360X.2017.00064.6.

40. Yadollahpour A, Jalilifar M, Rashidi S. Transcranial direct current stimulation for the treatment of depression: a comprehensive review of the recent advances. Int J Ment Health Addict. 2017. https://doi.org/10.1007/ s11469-017-9741-3.

41. Baeken C, Brunelin J, Duprat R, Vanderhasselt M-A. The application of tDCS in psychiatric disorders: a brain imaging view. Socioaffective Neurosci Psychol. 2016;6:29588. https://doi.org/10.3402/SNP.V6.29588.

42. Trumbo M. Effect of transcranial direct current stimulation on the attention network task (ANT). University of New Mexico. 2012. https://digit alrepository.unm.edu/psy_etds/139/. Accessed 10 June 2018

43. Lefaucheur J-P, Antal A, Ayache SS, Benninger DH, Brunelin J, Cogiamanian F, et al. Evidence-based guidelines on the therapeutic use of transcranial direct current stimulation (tDCS). Clin Neurophysiol. 2017. https://doi.org/10.1016/j.clinph.2016.10.087.

44. Nitsche MA, Paulus W. Excitability changes induced in the human motor cortex by weak transcranial direct current stimulation. J Physiol. 2000:527:633-9.

45. Fregni F, Marcondes R, Boggio PS, Marcolin MA, Rigonatti SP, Sanchez TG, et al. Transient tinnitus suppression induced by repetitive transcranial magnetic stimulation and transcranial direct current stimulation. Eur J Neurol. 2006;13:996-1001.

46. Vanneste S, Langguth B, De Ridder D. Do tDCS and TMS influence tinnitus transiently via a direct cortical and indirect somatosensory modulating effect? a combined TMS-tDCS and TENS study. Brain Stimul. 2011;4:24252. https://doi.org/10.1016/j.brs.2010.12.001.

47. Faber M, Vanneste S, Fregni F, De Ridder D. Top down prefrontal affective modulation of tinnitus with multiple sessions of tDCS of dorsolateral prefrontal cortex. Brain Stimul. 2012;5:492-8.

48. Shekhawat GS, Stinear CM, Searchfield GD. Transcranial direct current stimulation intensity and duration effects on tinnitus suppression. Neurorehabil Neural Repair. 2013;27:164-72. https://doi.org/10.1177/15459 68312459908.

49. Romero Lauro LJ, Rosanova M, Mattavelli G, Convento S, Pisoni A, Opitz A, et al. TDCS increases cortical excitability: direct evidence from TMS-EEG. Cortex. 2014;58:99-111. https://doi.org/10.1016/j.cortex.2014.05.003.

50. Varoli E, Pisoni A, Mattavelli GC, Vergallito A, Gallucci A, Mauro LD, et al. Tracking the effect of cathodal transcranial direct current stimulation on cortical excitability and connectivity by means of TMS-EEG. Front Neurosci. 2018;12:319. https://doi.org/10.3389/fnins.2018.00319.

51. Pellicciari MC, Brignani D, Miniussi C. Excitability modulation of the motor system induced by transcranial direct current stimulation: a multimodal approach. Neuroimage. 2013;83:569-80. https://doi.org/10.1016/j.neuro image.2013.06.076.

52. Roche N, Geiger M, Bussel B. Mechanisms underlying transcranial direct current stimulation in rehabilitation. Ann Phys Rehabil Med. 2015;58:2149. https://doi.org/10.1016/J.REHAB.2015.04.009.

53. Shekhawat GS, Stinear CM, Searchfield GD. Transcranial direct current stimulation intensity and duration effects on tinnitus suppression. Neurorehabil Neural Repair. 2013;27:164-72. https://doi.org/10.1177/15459 68312459908.

54. Forogh B, Mirshaki Z, Raissi GR, Shirazi A, Mansoori K, Ahadi T. Repeated sessions of transcranial direct current stimulation for treatment of chronic subjective tinnitus: a pilot randomized controlled trial. Neurol Sci. 2016;37:253-9. https://doi.org/10.1007/s10072-015-2393-9.

55. Shekhawat GS, Sundram F, Bikson M, Truong D, De Ridder D, Stinear $\mathrm{CM}$, et al. Intensity, duration, and location of high-definition transcranial 
direct current stimulation for tinnitus relief. Neurorehabil Neural Repair. 2016:30:349-59. https://doi.org/10.1177/1545968315595286.

56. Wang Y, Shen Y, Cao X, Shan C, Pan J, He H, et al. Transcranial direct current stimulation of the frontal-parietal-temporal area attenuates cue-induced craving for heroin. J Psychiatr Res. 2016;79:1-3. https://doi. org/10.1016/j.jpsychires.2016.04.001.

57. Baker JM, Rorden C, Fridriksson J. Using transcranial direct-current stimulation to treat stroke patients with aphasia. Stroke. 2010;41:1229-36.

58. Fröhlich F, Burrello TN, Mellin JM, Cordle AL, Lustenberger CM, Gilmore $\mathrm{JH}$, et al. Exploratory study of once-daily transcranial direct current stimulation (tDCS) as a treatment for auditory hallucinations in schizophrenia. Eur Psychiatry. 2016;33:54-60. https://doi.org/10.1016/j.eurps y.2015.11.005.

59. Vanneste S, De Ridder D. Bifrontal transcranial direct current stimulation modulates tinnitus intensity and tinnitus-distress-related brain activity. Eur J Neurosci. 2011;34:605-14.

60. Frank E, Schecklmann M, Landgrebe M, Burger J, Kreuzer P, Poeppl TB, et al. Treatment of chronic tinnitus with repeated sessions of prefrontal transcranial direct current stimulation: outcomes from an open-label pilot study. J Neurol. 2012;259:327-33.

61. De Ridder D, Vanneste $S$. EEG driven tDCS versus bifrontal tDCS for tinnitus. Front Psychiatry. 2012;3:84.

62. Garin P, Gilain C, Van Damme JP, de Fays K, Jamart J, Ossemann M, et al. Short- and long-lasting tinnitus relief induced by transcranial direct current stimulation. J Neurol. 2011;258:1940-8. https://doi.org/10.1007/ s00415-011-6037-6.

63. Shekhawat GS, Searchfield GD, Stinear CM. Randomized trial of transcranial direct current stimulation and hearing aids for tinnitus management. Neurorehabil Neural Repair. 2014;28:410-9. https://doi. org/10.1177/1545968313508655.

64. Mori T, Takeuchi N, Suzuki S, Miki M, Kawase T, Izumi S-I. Anodal transcranial direct current stimulation over the auditory cortex improved hearing impairment in a patient with brainstem encephalitis. J Int Med Res. 2016;44:760-4. https://doi.org/10.1177/0300060516630843.

65. Vanneste S, De Ridder D. Noninvasive and invasive neuromodulation for the treatment of tinnitus: an overview. Neuromodulation Technol Neural Interface. 2012;15:350-60. https://doi.org/10.111 1/j.1525-1403.2012.00447.x.

66. Yadollahpour A, Bayat A, Rashidi S, Saki N, Karimi M. Dataset of acute repeated sessions of bifrontal transcranial direct current stimulation for treatment of intractable tinnitus: a randomized controlled trial. Data Br. 2017;15:40-6.

67. Bayat A, Mayo M, Rashidi S, Saki N, Yadollahpour A. Repeated sessions of bilateral transcranial direct current stimulation on intractable tinnitus: a study protocol for a double-blind randomized controlled trial. F1000Research. 2018;7:317. https://doi.org/10.12688/f1000research.13558.1.

68. Shekhawat GS, Vanneste S. Optimization of transcranial direct current stimulation of dorsolateral prefrontal cortex for tinnitus: a non-linear dose-response effect. Sci Rep. 2018;8:8311. https://doi.org/10.1038/s4159 8-018-26665-1.

69. Pal N, Maire R, Stephan MA, Herrmann FR, Benninger DH. Transcranial direct current stimulation for the treatment of chronic tinnitus: a randomized controlled study. Brain Stimul. 2015;8:1101-7. https://doi. org/10.1016/j.brs.2015.06.014.

70. Teismann H, Wollbrink A, Okamoto H, Schlaug G, Rudack C, Pantev C. Combining transcranial direct current stimulation and tailor-made notched music training to decrease tinnitus-related distress-a pilot study. PLoS One. 2014;9:e89904. https://doi.org/10.1371/journ al.pone.0089904.

71. Kreuzer PM, Lehner A, Schlee W, Vielsmeier V, Schecklmann M, Poeppl $T B$, et al. Combined rTMS treatment targeting the anterior cingulate and the temporal cortex for the treatment of chronic tinnitus. Sci Rep. 2015;5:18028

72. Joos K, De Ridder D, Van de Heyning P, Vanneste S. Polarity specific suppression effects of transcranial direct current stimulation for tinnitus. Neural Plast. 2014;2014:1-8. https://doi.org/10.1155/2014/930860.

73. Vanneste S, De Ridder D. Bifrontal transcranial direct current stimulation modulates tinnitus intensity and tinnitus-distress-related brain activity. Eur J Neurosci. 2011;34:605-14. https://doi.org/10.111 1/j.1460-9568.2011.07778.x.

74. Smits M, Kovacs S, De Ridder D, Peeters RR, Van Hecke P, Sunaert S. Lateralization of functional magnetic resonance imaging (fMRI) activation in the auditory pathway of patients with lateralized tinnitus. Neuroradiology. 2007:49:669-79.

75. Schlee W, Mueller N, Hartmann T, Keil J, Lorenz I, Weisz N. Mapping cortical hubs in tinnitus. BMC Biol. 2009;7:80.

76. Wagner BT. Non invasive brain stimulation : modeling and experimental analysis of transcranial magnetic stimulation and transcranial DC stimulation as a modality for neuropathology treatment technology. Cambridge: Harvard-MIT Division of Health Sciences And Tec; 2006.
Ready to submit your research? Choose BMC and benefit from:

- fast, convenient online submission

- thorough peer review by experienced researchers in your field

- rapid publication on acceptance

- support for research data, including large and complex data types

- gold Open Access which fosters wider collaboration and increased citations

- maximum visibility for your research: over $100 \mathrm{M}$ website views per year

At BMC, research is always in progress.

Learn more biomedcentral.com/submissions 